\title{
Detection and characterization of human astrovirus and sapovirus in outpatients with acute gastroenteritis in Guangzhou, China
}

\author{
Xin Luo ${ }^{1}$, Jian-kai Deng ${ }^{2}$, Xiao-ping $\mathrm{Mu}^{1}$, $\mathrm{Nan} \mathrm{Yu}^{3 *}$ and Xiaoyan $\mathrm{Che}^{3 \wedge}$
}

\begin{abstract}
Background: Human astrovirus (HAstV) and sapovirus (SaV) are common pathogens that can cause acute gastroenteritis (AGE). However, very few studies have reported the molecular epidemiology and clinical information on HAstV and SaV in China. This study aims to determine the molecular epidemiology and clinical features of HAstV and SaV in patients with AGE in Guangzhou, China.

Methods: For this study, 656 patients with AGE were enrolled. Their stool samples were screened for 15 enteropathogens using Luminex $\times \mathrm{XTG}^{\circledR}$ Gastrointestinal Pathogen Panel. HAstV and SaV were detected through an in-house multiplex reverse transcriptase polymerase chain reaction followed by phylogenetic analysis. We described and compared clinical features of AGE in patients with HAstV and SaV.

Results: Of the 656 stool samples, $63.72 \%$ (418/656) were found to be positive, with 550 enteropathogens (296 bacteria and 254 viruses). HAstV and SaV were detected in 20 (3.0\%) and 12 (1.8\%) samples, respectively. Four genotypes (genotypes 1, 2, 3, and 8) of HAstV and three genotypes (GI.1, GI.2 and GIV) of SaV were identified. Coinfection was observed in ten HAstV-positive and two SaV-positive samples. HAstV was more likely to occur in winter, while SaV in early spring. The median age of the patients with single HAstV infection was higher than that of the patients with other viruses (rotavirus, norovirus, and enteric adenovirus; $P=0.0476)$ and unknown etiology $(P=0.006)$. Coinfection with HAstV or SaV were not associated with disease severity $(P>0.05)$.
\end{abstract}

Conclusion: HAstV and SaV are the common causes of AGE in Guangzhou, China.

Keywords: Acute gastroenteritis, Astrovirus, Sapovirus, Clinical features, Molecular epidemiology

\section{Background}

Acute gastroenteritis (AGE) is a common illness of humans globally. It adversely affects the public health, especially the very young, the elderly, the malnourished, and those with an impaired immune system [13]. Human AGE is caused by a spectrum of viruses and

\footnotetext{
*Correspondence: hardroom@126.com

${ }^{3}$ Laboratory of Emerging Infectious Diseases and Division of Laboratory Medicine, Zhujiang Hospital, Southern Medical University, No. 253 Gong-ye Avenue, Guangzhou 510282, People's Republic of China Full list of author information is available at the end of the article Xiaoyan Che: Deceased
}

bacteria. Viruses, including norovirus, rotavirus, enteric adenovirus, astrovirus (HAstV), and sapovirus $(\mathrm{SaV})$, are the major causative agents for AGE $[4,5]$. Although detailed epidemiological data for both domestic and overseas infectious gastroenteritis caused by rotavirus, norovirus, and enteric adenovirus is available $[6,7]$, little is known about the clinical symptoms, characteristics, and coinfection of HAstV- and SaV-related AGE because of the various limitations of the detection methods and low detection rates. Further epidemiological and molecular information about HAstV and SaV causing AGE may prove useful in the development of $\mathrm{HAstV} / \mathrm{SaV}$ vaccines 
and other preventative therapies for AGE in the post rotavirus-vaccination introduction era.

The present study aims to determine the incidence of HAstV and $\mathrm{SaV}$ in outpatients with AGE in Guangzhou, China, and characterize HAstV and $\mathrm{SaV}$ according to their genotype, age-related distribution, seasonal pattern and clinical symptoms.

\section{Methods}

\section{Sample collection}

From September 2013 to January 2016, 656 stool samples were collected from 656 outpatients who were diagnosed with AGE. The definition of AGE was the sudden onset of $>3$ episodes of diarrhea or vomiting in the preceding $24 \mathrm{~h}$ and symptom duration $<7$ days. All of the enrolled stool samples were routinely collected and stored at $-70{ }^{\circ} \mathrm{C}$ prior to investigation. The stool samples were preprocessed using NucliSENS easyMAG Lysis Buffer (BIOMERIEUX, France), followed by Viral RNA and DNA extraction using QIAamp MinElute Virus Spin kit (QIAGEN, Germany), as described previously [8].

\section{Multiplex RT-PCR and nucleotide sequencing}

In-house multiplex RT-PCR was developed to detect HAstV, SaV, norovirus, and enteric adenovirus. The primers used for the amplification and sequencing for HAstV (target ORF1a gene), SaV (target ORF1 gene), norovirus GI/GII (target ORF2 gene), and enteric adenovirus (target Hexon gene) were obtained from the published literature [9-12]. The primers used were formulated by Sangon Biotech (Shanghai, China). The product sizes were $482 \mathrm{bp}$ (enteric adenovirus), $288 \mathrm{bp}$ (HAstV), 434 bp (SaV), 330 bp (norovirus GI), and 387 bp (norovirus GII). The PCR was performed on a Veriti PCR (Life Technologies, USA) using the PrimeScript One Step RT-PCR Kit (TaKaRa, Japan). Briefly, $25 \mu \mathrm{L}$ of the reaction mix comprised Enzyme Mix $(1.0 \mu \mathrm{L})$, buffer $(12.5 \mu \mathrm{L})$, RNase-free water $(1.0 \mu \mathrm{L})$, primers $(20 \mu \mathrm{mol} / \mathrm{L}$ each $)$, and RNA/DNA extraction $(5.0 \mu \mathrm{L})$. Reverse transcription was carried out at $50{ }^{\circ} \mathrm{C}$ for $30 \mathrm{~min}$, followed by predenaturation at $94{ }^{\circ} \mathrm{C}$ for $3 \mathrm{~min}, 94{ }^{\circ} \mathrm{C} 30 \mathrm{~s}, 54{ }^{\circ} \mathrm{C} 30 \mathrm{~s}$, and $72{ }^{\circ} \mathrm{C} 45 \mathrm{~s}$ for 40 cycles with a final extension at $72{ }^{\circ} \mathrm{C}$ for $12 \mathrm{~min}$. The amplified viral nucleic acid product was detected by $2 \%$ agarose gel electrophoresis. Multiplex RT-PCR products that were positive for $\mathrm{HAstV}$ and $\mathrm{SaV}$ were sequenced by a commercial service (BGI Tech Corporation, Shenzhen, China).

\section{xTAG gastrointestinal pathogen panel assay}

Following the manufacturer's protocol, the xTAG Gastrointestinal Pathogen Panel (xTAG GPP) assay was used to detect the genes of 15 enteropathogens-Salmonella spp., Shigella spp., Campylobacter spp., Yersinia. enterocolitica, Vibrio cholerae, Escherichia. coli O157, enterotoxigenic E. coli (ETEC) LT/ST, Shiga-like toxinproducing E. coli (STEC) stx1/stx2, Clostridium difficile toxins $\mathrm{A} / \mathrm{B}$, rotavirus $\mathrm{A}$, enteric adenovirus $40 / 41$, norovirus GI/GII, Cryptosporidium spp., Entamoeba histolytica, and Giardia lamblia.

\section{Phylogenetic analysis}

The obtained sequences of the HAstV- and SaV-positive samples were aligned by Clustal X (version 5.0). Phylogenetic tree was constructed by the neighbor-joining method using MEGA 6.0 software. The genetic distance was calculated using the Kimura 2-Parameters model with 1000 bootstrap resamples of the nucleotide alignment for genotypic strain classification. The following reference strains and accession numbers were used: L23513, L13745, AF141381, KF039913.1, JQ403108, GQ901902, AF290508, AF260508, FJ973620, GQ502193, FJ402983, GQ502192, NC_019028, and NC_013443 for HAstV; and U65427, X86560, U95643, AB518056, U73124, U95644, AB614356, HM800913, HUN3739, KJ826502, AY603425, AY237420, U95645, AJ249939, AF182760, AY425671, AF435814, DQ125333, and AY64685 for SaV. The nucleotide sequence data reported herein have been published in the GenBank nucleotide sequence database under the accession numbers of HAstV and $\mathrm{SaV}$ isolates: KP090035-KP090047, KR364727-KR364729, KX522566-KX522573, KT751010-KT751011, and KX530323-KX530328.

\section{Statistical analysis}

All statistical analyses were performed using SPSS version 21.0 (SPSS Inc., Chicago, IL, USA). Differences among the proportions were compared by the Chisquare Test, Fisher's Exact Test, or Mann-Whitney U Test. Differences with $P<0.05$ were considered statistically significant.

\section{Results}

Distribution of enteropathogens in patients with AGE

A total of 656 stool samples were enrolled in the present study. The age distribution of the patients varied from 9 days to 84 years, with the median age of 25 months. Out of the 656 patients, 387 (59.0\%) were male and $521(79.4 \%)$ were under 5 years old. We detected 418 (63.72\%) positive samples, resulting in 550 enteropathogens (296 bacteria and 254 viruses). HAstV and $\mathrm{SaV}$ were detected in $20(3.0 \%)$ and $12(1.8 \%)$ samples, respectively, while rotavirus was detected in 112 (17.1\%), norovirus in 103 (15.7\%), and enteric adenovirus in 7 (1.1\%) samples. Norovirus GI and GII and adenovirus were detected by both the xTAG GPP and the in-house multiplex RT-PCR (Kappa value was 0.884, 
0.918 , and 0.791 , respectively, showing high consistency between xTAG GPP and the in-house multiplex RT-PCR) [13].

Among people with AGE who tested positive for HAstV, $50 \%(10 / 20)$ had one or more codetected enteropathogens: there are one other pathogen in $40 \%(8 / 20)$, and there are two other pathogens in $10 \%$ $(2 / 20) .16 .7 \%(2 / 12)$ of $\mathrm{SaV}$ positive samples had one or more codetected enteropathogens: there are one other pathogen in $0.2 \%(1 / 12)$, and there are three other pathogens in $0.2 \%$ (1/12). Rotavirus, Norovirus, Clostridium difficile, Salmonella, Campylobacter were the common codetected enteropathogens. (Table 1).

Table 1 Coinfection with HAstV and SaV among people with acute gastroenteritis in Guangzhou, China from 2013 to 2016

\begin{tabular}{ll}
\hline Infection or coinfection & $\begin{array}{l}\text { No. (\%) of AGE } \\
\text { cases ( } \mathbf{n = 6 5 6 )}\end{array}$ \\
\hline HAstV alone & $10(1.5)$ \\
SaV alone & $10(1.5)$ \\
2 pathogens codetected & \\
HAstV, rotavirus & $3(0.5)$ \\
HAstV, norovirus & $3(0.5)$ \\
HAstV, Clostridium difficile & $1(0.2)$ \\
HAstV, Salmonella & $1(0.2)$ \\
SaV, norovirus & $1(0.2)$ \\
3 pathogens codetected & \\
HAstV, Clostridium difficile, Norovirus & $1(0.2)$ \\
HAstV, rotavirus, Campylobacter, & $1(0.2)$ \\
4 pathogens codetected & $1(0.2)$ \\
SaV, Campylobacter, rotavirus, Salmonella &
\end{tabular}

\section{Seasonal and age distribution of HAstV and SaV infection} in people with $\mathrm{AGE}$

Seasonal variation in $\mathrm{HAstV}$ and $\mathrm{SaV}$ infection is shown in Fig. 1 . The monthly mean temperature for the period 2013-2016 in Guangzhou is also shown in Fig. 1. HAstV was detected most commonly in February $(8.33 \%, 3 / 36)$. The peak of SaV was March (15.0\%, 3/20).

Age distribution in HAstV and $\mathrm{SaV}$ infections is shown in Fig. 2. HAstV showed the highest detection rate in 15-59-year age group (11.9\%), follow by $1-5$-year age group (5.97\%). SaV infection was most common in 1-5-year age group (5.97\%). HAstV and $\mathrm{SaV}$ were not detected in the 5-14-year-old age group. The median age of people with AGE due to single HAstV infection was higher than that of people with other viruses (rotavirus, norovirus, and enteric adenovirus) $(P=0.0476)$ and unknown etiology $(P=0.006)$ (Table 2$)$.

\section{Clinical features of HAstV and SaV infections in people with AGE}

Single SaV infections and infections by other viruses or unknown etiology did not have much different clinical features such as fever, vomit, diarrheal frequency, and stool routine examination (WBC count and $\mathrm{OB}$ text). However, AGE with single HAstV infection is more likely to cause fever and has been observed in five of the ten cases (50\%) compared to infections caused by unknown etiology $(16.81 \%)(P=0.0197)$. No significant difference was observed in the incidence of vomit, diarrheal frequency, and stool routine examination (WBC count and OB text) between single HAstV infection and other virus infections or infections by unknown etiology. The median (IQR) of diarrheal frequency (times/day) in patients with single HAstV,

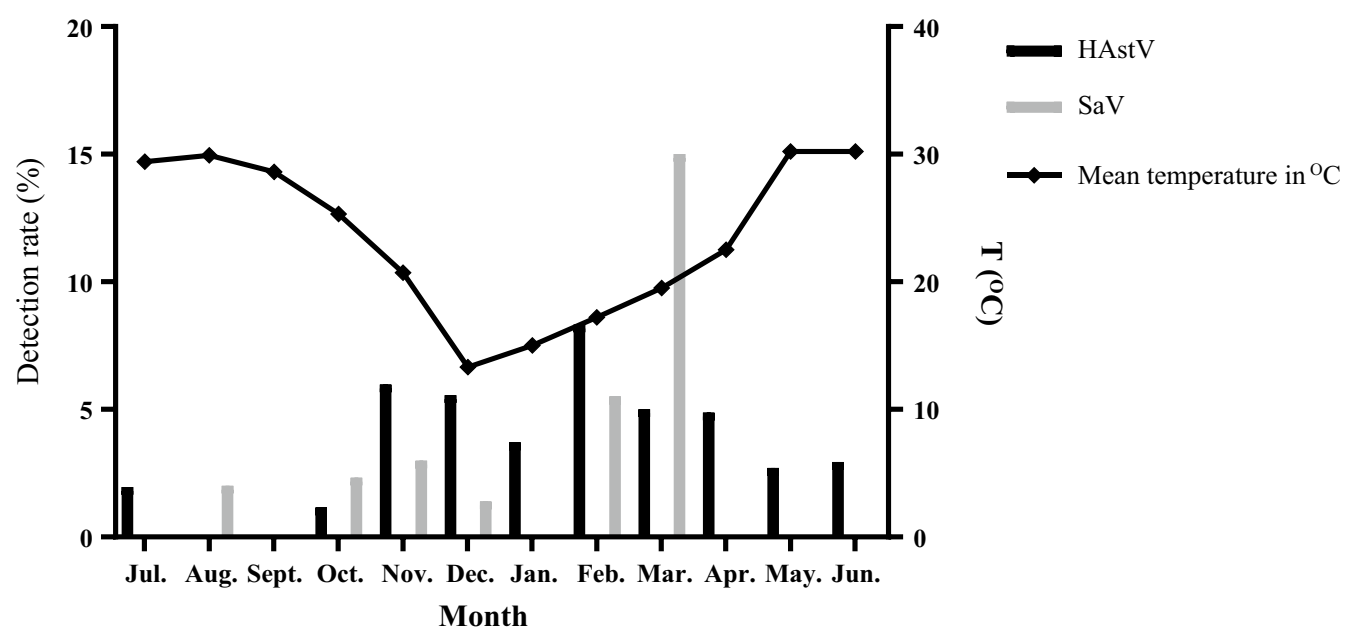

Fig. 1 Monthly distribution of HAstV and SaV in people with acute gastroenteritis 


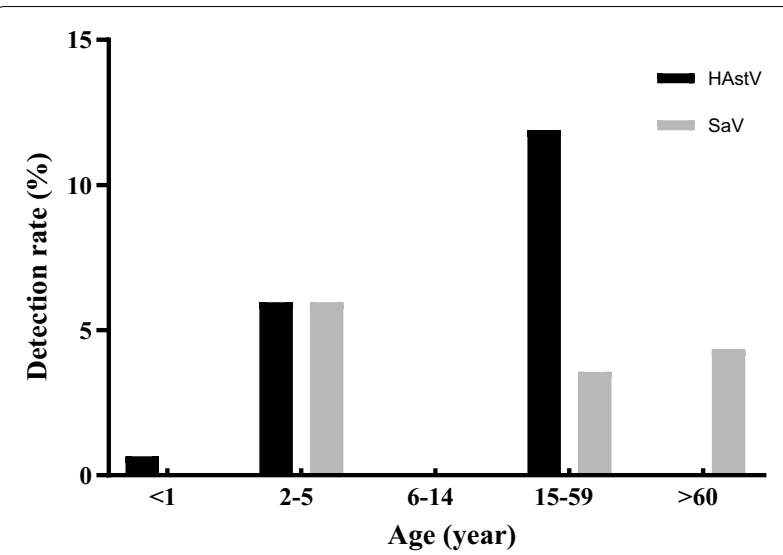

Fig. 2 Age distribution of HAstV and SaV in people with acute gastroenteritis

single $\mathrm{SaV}$, and other virus infections were $6.5(4.75$, $8.50), 3.50(3.00,7.00)$, and $5.00(4.00,8.00)$, although the difference was not statistically significant. On other hand, we did not find any difference in the clinical characteristics of patients with single $\mathrm{HAstV} / \mathrm{SaV}$ infections and those showing coinfection with HAstV/ $\mathrm{SaV}$ (Table 2).
Nucleotide sequencing and phylogenetic analysis of HAstV and SaV

A total of $20 \mathrm{HAstV}$ and $12 \mathrm{SaV}$ sequences were analyzed by phylogenetic analysis. Four genotypes (genotypes 1 , 2, 3, and 8) of HAstV and three genotypes (GI.1, GI.2 and GIV) of SaV were identified (Fig. 3). HAstV-2 (35\%, $7 / 20$ ) was the dominant genotype, followed by HAstV-1 $(30 \%, 6 / 20)$, HAstV-8 $(20 \%, 4 / 20)$ and HAstV-3 (15\%, $3 / 20)$. SaV GI.1 had the highest constituent ratio at $58.3 \%$ (7/12), followed by SaV GI.2 $(33.3 \%, 4 / 12)$ and SaV GIV $(8.3 \%, 1 / 12)$.

\section{Discussion}

AGE is one of the most common disease around the world. However, other than rotavirus, norovirus and enteric adenovirus, knowledge about the impact of infections caused by HAstV and $\mathrm{SaV}$ is still much needed. In an effort to understand better the role of $\mathrm{HAstV}$ and $\mathrm{SaV}$ as a cause of AGE, a comprehensive survey on HAstV and $\mathrm{SaV}$ was carried out in Guangzhou, China. In-house multiplex RT-PCR and xTAG GPP, which had been validated for enteropathogens detection ability before, were used to detect HAstV, $\mathrm{SaV}$ and other enteropathogens associated with AGE $[8,14]$.

The detection rate of HAstV in people with AGE was $3.0 \%$ in the present study, which is similar to that previously reported in shanghai $(5.22 \%)$, Thailand (2.6\%), Asian Russia (2.8\%) and Germany (5.0\%), but was lower

Table 2 Comparison of clinical characteristics in the HAstV and SaV infection among people with acute gastroenteritis in Guangzhou, China from 2013 to 2016

\begin{tabular}{|c|c|c|c|c|c|c|}
\hline Variables & $\begin{array}{l}\text { Single HAstV } \\
\text { infection }(n=10)\end{array}$ & $\begin{array}{l}\text { Coinfection with } \\
\text { HAstV }(n=10)\end{array}$ & $\begin{array}{l}\text { Single SaV } \\
\text { infection }(n=10)\end{array}$ & $\begin{array}{l}\text { Coinfection } \\
\text { with SaV }(n=2)\end{array}$ & $\begin{array}{l}\text { Other virus } \\
\text { infection } \\
(n=202)\end{array}$ & $\begin{array}{l}\text { Unknown } \\
\text { etiology cases } \\
(n=238)\end{array}$ \\
\hline \multicolumn{7}{|l|}{ Demographics } \\
\hline Male, $n(\%)$ & $6(60)$ & $6(60)$ & $3(30)$ & $2(100)$ & $124(61.39)$ & $145(60.92)$ \\
\hline Age range (year) & 0.92 to 49 & 0.03 to 42 & 1 to 72 & 1.17 to 2 & 0.08 to 81 & 0.08 to 84 \\
\hline Median (IQR) & $11.00(1.00,27.50)^{*}$ & $15.50(1.23,32.75)$ & $2.50(1.33,25.00)$ & $1.59(1.17,2.00)$ & $1.00(0.92,3.00)$ & $0.67(0.42,1.00)$ \\
\hline \multicolumn{7}{|l|}{ Clinical presentations } \\
\hline Fever $>37.5^{\circ} \mathrm{C}, n(\%)$ & $5(50)^{* *}$ & $1(10)$ & $1(10)$ & $0(0)$ & $66(32.67)$ & $40(16.81)$ \\
\hline Vomit, $n(\%)$ & $2(20)$ & $5(50)$ & $2(20)$ & $0(0)$ & $97(48.02)$ & $30(12.61)$ \\
\hline Diarrhea, $n(\%)$ & $10(100)$ & $10(100)$ & $10(100)$ & $2(100)$ & $202(100)$ & $238(100)$ \\
\hline $\begin{array}{l}\text { Frequency (times/day), } \\
\text { median (IQR) }\end{array}$ & $6.50(4.75,8.50)$ & $4.50(3.00,6.25)$ & $3.50(3.00,7.00)$ & $3.00(3.00,3.00)$ & $5.00(4.00,8.00)$ & $5.00(4.00,8.00)$ \\
\hline \multicolumn{7}{|l|}{ Laboratory findings in stool } \\
\hline WBC count (/HP), n (\%) & $2(20)$ & $4(40)$ & $1(10)$ & $0(0)$ & $58(28.71)$ & $78(32.77)$ \\
\hline OB positive, $n(\%)$ & $4(40)$ & $2(20)$ & $2(20)$ & $0(0)$ & $49(24.26)$ & $73(30.67)$ \\
\hline
\end{tabular}

Unknown etiology cases: no enteric pathogens were detected

Other virus infection: includes rotavirus, norovirus, and enteric adenovirus infection, but excludes coinfection with HAstV and SaV

$O B$ stool occult blood test

${ }^{*} P<.05$ compared to other virus infection and unknown etiology cases

${ }^{*} P<.05$ comspared to unkown etiology cases 


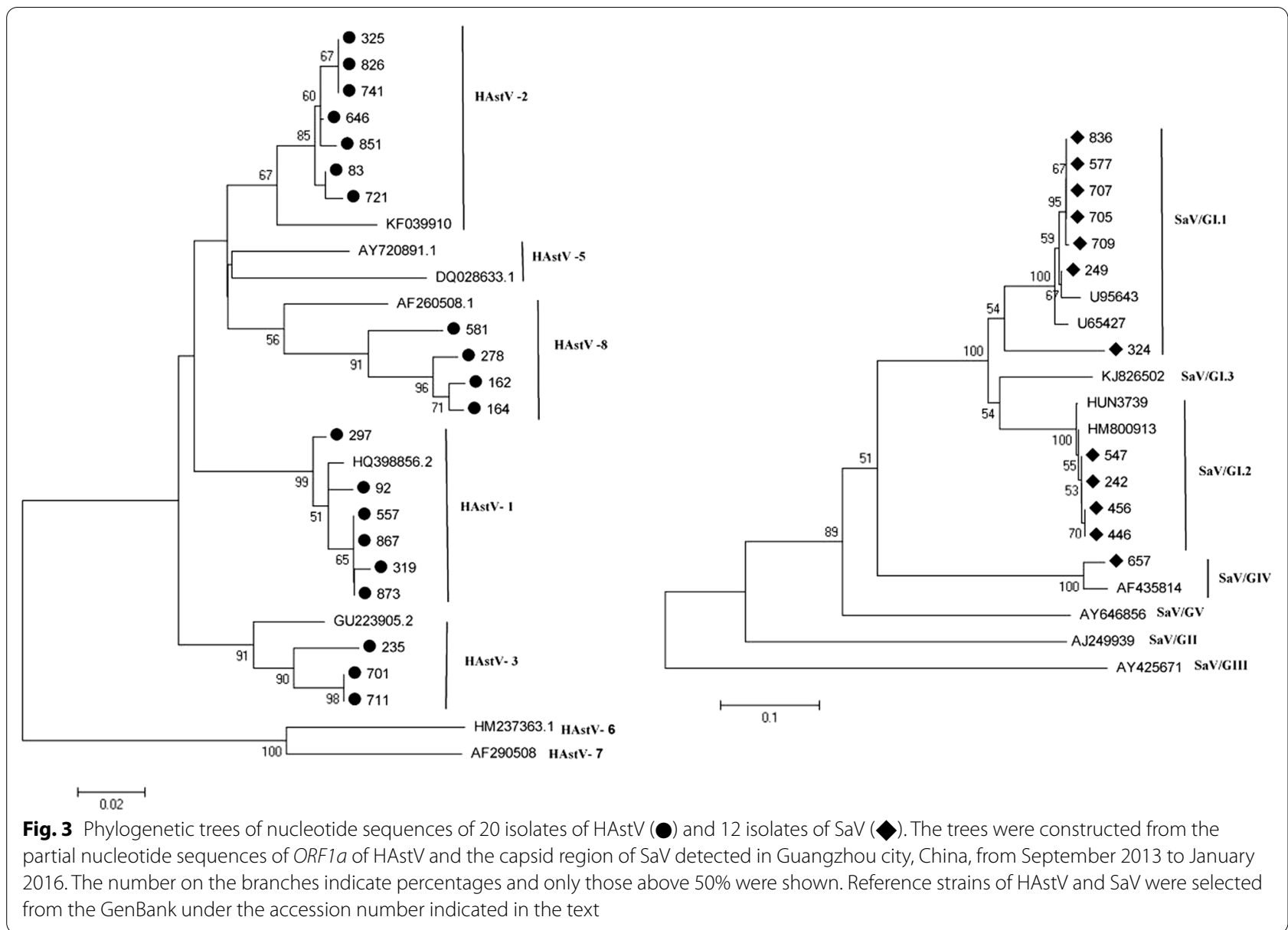

than the mean incidence worldwide of $11.0 \%$ [13, 15-18]. The SaV positive rate was $1.8 \%$ in Guangzhou, which is lower than that observed in US (7.0\%) and Canada (9.5\%) $[19,20]$. Coinfection was found in $50 \%$ of the HAstVpositive samples and $16.7 \%$ of the $\mathrm{SaV}$-positive samples in our study. Using real-time RT-PCR, other studies have reported a higher rate of coinfection with HAstV in viral gastroenteritis, ranging between 77 and $80 \%$ [21, 22]. Zhuo et al. reported a $35 \%$ prevalence of coinfection with $\mathrm{SaV}$ in Western Canada over a 4-year period [20]. Our slightly lower positivity can be explained by differences in the sample size, geographic location, and detection method. Norovirus and rotavirus were the most frequently detected enteropathogens responsible for coinfection in our study, which is in agreement with earlier reports [21-24]. It can be suggested that coinfection with HAstV or $\mathrm{SaV}$ is not rare. Our data also confirmed HAstV and $\mathrm{SaV}$ as the common enteropathogens responsible for AGE in Guangzhou. However, PCR can pick up free nucleic acid or long-term intermittent and asymptomatic shedding of $\mathrm{HAstV}, \mathrm{SaV}$ or other enteropathogens in patients. A positive detection does not always correspond to active infection. Further investigation of the viral load and pathogenic mechanism is required.

$\mathrm{SaV}$ was detected most commonly in 1-5-year age group (5.97\%), which is in line with those reported previously, ie most of the HAst $\mathrm{V}, \mathrm{SaV}$, rotavirus, norovirus, and enteric adenovirus infections occurred predominantly in infants and children $<5$ years of age [21-24]. However, in contrast to the studies above, the prevalence of HAstV was the highest in 15-59-year age group $(11.90 \%)$ in this study. Our data also showed that the median age of patients with the single HAstV infection was higher than that of the patients with other virus infections $(P=0.0476) .10$ of total 20 cases with HAstV infection reported in 15-59-year age group, suggesting that adult may be susceptible to HAstV, not just the very young.

December to February the following year is the winter season in China. In our study, the highest incidence of HAstV occurs in winter (February) and that of SaV in early spring (March). Similar to several other studies of viral gastroenteritis conducted in India, Australia, Italy and Shanghai, HAstV infection was more frequent 
during the cold weather period in Guangzhou [18, 23, 25, 26]. SaV infection has been found mainly in the cold season $[20,27,28]$. However, the present study showed that $\mathrm{SaV}$ infection was more common during winter to early spring (February to March) in Guangzhou.

HAstV and $\mathrm{SaV}$ infections cause low-to-moderate degree of AGE with vomiting, fever, anorexia, abdominal pain, and dehydration $[13,29]$. In our study, all HAstVand $\mathrm{SaV}$-positive cases were associated with acute watery gastroenteritis. Fever and vomiting were reported in 30\% and $35 \%$ of HAstV infections and in $8.3 \%$ and $16.6 \%$ of $\mathrm{SaV}$ infections, respectively. In contrast to the study that reported greater diarrhea and fever prevalence, longer duration and greater intensity of diarrhea in HAstV infection compared to that in norovirus infections, our data did not show any significant difference in diarrhea frequency, fever prevalence between single HAstV infection and other virus infections [30]. Some reports suggest that coinfection may lead to more severe diarrhea [31, 32]. However, we could not find any significant difference in the specific clinical severity of AGE between single $\mathrm{HAstV} / \mathrm{SaV}$ infections and mixed HAstV/SaV infections, which is in accordance with the reports from India and other countries [30, 33, 34]. In one case of Campylobacter, Salmonella, rotavirus, and SaV coinfection, no fever or vomiting was observed; in addition, the test for the presence of white/red blood cell test in the stool was negative, with low degree of gastroenteritis symptoms.

HAstV-1 is the most common HAstV genotype circulating worldwide [35-37]. However, predominant genotypes may change in different geographical locations, as has been reported in cases such as HAstV-2-associated childhood viral gastroenteritis outbreaks in Colombia [38]. In our study, six cases of HAstV-1, seven cases of HAstV-2, three cases of HAstV-3, and four cases of HAstV-8 were noted. HAstV-1 and HAstV-2 are predominant genotypes of HAstV in Guangzhou. SaV GI is the most common genogroup, while SaV GI.1 is the most common genotype globally [15, 24, 39]. In our study, 91.7\% of the detected $\mathrm{SaV}$ cases belonged to GI, which is in accordance with literature. The most prevalent $\mathrm{SaV}$ genotype was GI.1 (58.4\%), followed by GI.2 (33.3\%) and GIV (8.3\%).

\section{Conclusion}

Although this study has established HAstV and $\mathrm{SaV}$ as the common causes of AGE in Guangzhou, the true etiologic agent could not be determined with certainty because of the high proportion of coinfection. Hence, further investigation of the viral load or unrecognized agents is required. The results of this study may provide further epidemiological and molecular information about HAstV and $\mathrm{SaV}$ strains causing AGE.
Acknowledgements

We thank the participants for their contributions to the study. The authors greatly benefitted from the research studies, mentorship, and friendship of the late Dr Xiaoyan Che.

\section{Authors' contributions}

$\mathrm{XL}, \mathrm{XYC}$ and $\mathrm{NY}$ conceived and designed the study. XL, JKD and NY supported in screening tests for viral agent detection and genomic sequencing. $X L$ and NY participated in data analysis. XL, JKD, XPM and NY participated in the drafting of the article and the performance of the research. All authors read and approved the final manuscript.

\section{Funding}

This work was supported by grant 2012ZX10004-213 and 2014ZX10004005 of the National Projects of Major Infectious Disease Control and Prevention.

\section{Availability of data and materials}

The datasets analysed during the current study available from the corresponding author on reasonable request.

\section{Declarations}

\section{Ethics approval and consent to participate}

This study was approved by Ethical committee of Guangdong Women and Children Hospital (Approval number: 202101280), all methods were carried out in accordance with relevant guidelines and regulations. The ethical committee of Guangdong Women and Children Hospital has determined the fecal specimens enrolled in this study were left over after routine examination, so no informed consent was obtained.

\section{Consent for publication \\ Not applicable.}

\section{Competing interests}

The authors declare that there are no competing interests.

\section{Author details}

${ }^{1}$ Department of Laboratory Medicine, Guangdong Women and Children Hospital, Guangzhou 511400, China. ${ }^{2}$ Department of Laboratory Medicine, The First Affiliated Hospital, Sun Yat-sen University, Guangzhou 510080, China. ${ }^{3}$ Laboratory of Emerging Infectious Diseases and Division of Laboratory Medicine, Zhujiang Hospital, Southern Medical University, No. 253 Gong-ye Avenue, Guangzhou 510282, People's Republic of China.

Received: 28 June 2021 Accepted: 25 November 2021

Published online: 03 December 2021

\section{References}

1. Liu L, Oza S, Hogan D, Perin J, Rudan I, Lawn JE, Cousens S, Mathers C, Black RE. Global, regional, and national causes of child mortality in 2000-13, with projections to inform post-2015 priorities: an updated systematic analysis. Lancet. 2015;385(9966):430-40. https://doi.org/10. 1016/S0140-6736(14)61698-6.

2. Lopez AD, Mathers CD, Ezzati M, Jamison DT, Murray CJ. Global and regional burden of disease and risk factors, 2001: systematic analysis of population health data. Lancet. 2006;367(9524):1747-57. https://doi.org/ 10.1016/S0140-6736(06)68770-9.

3. Liu L, Oza S, Hogan D, Chu Y, Perin J, Zhu J, Lawn JE, Cousens S, Mathers C, Black RE. Global, regional, and national causes of under-5 mortality in 2000-15: an updated systematic analysis with implications for the sustainable development goals. Lancet. 2016;388(10063):3027-35. https:// doi.org/10.1016/S0140-6736(16)31593-8.

4. Taco-Masias AA, Fernandez-Aristi AR, Cornejo-Tapia A, Aguilar-Luis MA, Del Valle L, Silva-Caso W, Zavaleta-Gavidia V, Weilg P, Cornejo-Pacherres H, Bazán-Mayra J, Puyen ZM, Del Valle-Mendoza J. Gut microbiota in hospitalized children with acute infective gastroenteritis caused by virus or bacteria in a regional Peruvian hospital. PeerJ. 2020;8:e9964. https:// doi.org/10.7717/peerj.9964. 
5. Amaral MS, Estevam GK, Penatti M, Lafontaine R, Lima IC, Spada PK, Gabbay YB, Matos NB. The prevalence of norovirus, astrovirus and adenovirus infections among hospitalised children with acute gastroenteritis in Porto Velho, state of Rondônia, western Brazilian Amazon. Mem Inst Oswaldo Cruz. 2015;110(2):215-21. https://doi.org/10.1590/0074-02760140381.

6. Hartman S, Brown E, Loomis E, Russell HA. Gastroenteritis in children. Am Fam Phys. 2019;99(3):159-165. Erratum in: Am Fam Phys. 2019;99(12):732.

7. Lee RM, Lessler J, Lee RA, Rudolph KE, Reich NG, Perl TM, Cummings DA. Incubation periods of viral gastroenteritis: a systematic review. BMC Infect Dis. 2013;13:446. https://doi.org/10.1186/1471-2334-13-446.

8. Deng J, Luo X, Wang R, Jiang L, Ding X, Hao W, Peng Y, Jiang C, Yu N, Che $X$. A comparison of Luminex XTAG ${ }^{\circledR}$ Gastrointestinal Pathogen Panel (XTAG GPP) and routine tests for the detection of enteropathogens circulating in Southern China. Diagn Microbiol Infect Dis. 2015;83(3):325-30. https://doi.org/10.1016/j.diagmicrobio.2015.07.024.

9. Yan H, Yagyu F, Okitsu S, Nishio O, Ushijima H. Detection of norovirus (GI, GII), sapovirus and astrovirus in fecal samples using reverse transcription single-round multiplex PCR. J Virol Methods. 2003;1 14(1):37-44. https:// doi.org/10.1016/j.jviromet.2003.08.009.

10. Yan H, Nguyen TA, Phan TG, Okitsu S, Li Y, Ushijima H. Development of RTMultiplex PCR assay for detection of enteric adenovirus and group A and C rotaviruses in diarrheal fecal specimens from children in China. J Jpn Assoc Infect Dis. 2004;78:699-709. https://doi.org/10.11150/kansenshog akuzasshi1970.78.699.

11. Kojima S, Kageyama T, Fukushi S, Hoshino FB, Shinohara M, Uchida K, Natori K, Takeda N, Katayama K. Genogroup-specific PCR primers for detection of Norwalk-like viruses. J Virol Methods. 2002;100(1-2):107-14. https://doi.org/10.1016/s0166-0934(01)00404-9.

12. Belliot $\mathrm{G}$, Laveran $\mathrm{H}$, Monroe SS. Detection and genetic differentiation of human astroviruses: phylogenetic grouping varies by coding region. Arch Virol. 1997;142(7):1323-34. https://doi.org/10.1007/s007050050163.

13. Johnson C, Hargest V, Cortez V, Meliopoulos VA, Schultz-Cherry S. Astrovirus pathogenesis. Viruses. 2017;9(1):22. https://doi.org/10.3390/v9010022.

14. Xin L, Nan Y, Yonghui G, Jiankai D, Xixia D, Ruilian W, Ning F, Xiaoyan C. Compare a multiplex RT-PCR method with liquichip technology in the detection of diarrhea-related virus, China. J Lab Med. 2015;38:387-91. https://doi.org/10.3760/cma.jissn.1009-9158.2015.06.007.

15. Kumthip K, Khamrin P, Ushijima H, Maneekarn N. Molecular epidemiology of classic, MLB and VA astroviruses isolated from $<5$ year-old children with gastroenteritis in Thailand, 2011-2016. Infect Genet Evol. 2018;65:373-9. https://doi.org/10.1016/j.meegid.2018.08.024.

16. Zhirakovskaia E, Tikunov A, Tymentsev A, Sokolov S, Sedelnikova D, Tikunova N. Changing pattern of prevalence and genetic diversity of rotavirus, norovirus, astrovirus, and bocavirus associated with childhood diarrhea in Asian Russia, 2009-2012. Infect Genet Evol. 2019;67:167-82. https://doi.org/10.1016/j.meegid.2018.11.006.

17. Jacobsen S, Höhne M, Marques AM, BesImüller K, Bock CT, Niendorf S Co-circulation of classic and novel astrovirus strains in patients with acute gastroenteritis in Germany. J Infect. 2018;76(5):457-64. https://doi.org/10. 1016/j.jinf.2018.02.006.

18. Lu L, Zhong H, Xu M, Su L, Cao L, Jia R, Xu J. Molecular and epidemiological characterization of human adenovirus and classic human astrovirus in children with acute diarrhea in Shanghai, 2017-2018. BMC Infect Dis. 2021;21(1):713. https://doi.org/10.1186/s12879-021-06403-1.

19. Hassan F, Kanwar N, Harrison CJ, Halasa NB, Chappell JD, Englund JA, Klein EJ, Weinberg GA, Szilagyi PG, Moffatt ME, Oberste MS, Nix WA, Rogers S, Bowen MD, Vinjé J, Wikswo ME, Parashar UD, Payne DC, Selvarangan R. Viral Etiology of acute gastroenteritis in < 2-year-old US children in the post-rotavirus vaccine era. J Pediatr Infect Dis Soc. 2019;8(5):414-21. https://doi.org/10.1093/jpids/piy077.

20. Zhuo R, Ding X, Freedman SB, Lee BE, Ali S, Luong J, Xie J, Chui L, Wu Y, Pang X. Alberta Provincial Pediatric EnTeric Infection TEam (APPETITE). Molecular epidemiology of human sapovirus among children with acute gastroenteritis in Western Canada. J Clin Microbiol. 2021;59(10):e0098621. https://doi.org/10.1128/JCM.00986-21.

21. Bergallo M, Galliano I, Daprà V, Rassu M, Montanari P, Tovo PA. Molecular detection of human astrovirus in children with gastroenteritis, Northern Italy. Pediatr Infect Dis J. 2018;37(8):738-42. https://doi.org/10.1097/INF. 0000000000001899 .

22. Olortegui MP, Rouhani S, Yori PP, Salas MS, Trigoso DR, Mondal D, Bodhidatta L, Platts-Mills J, Samie A, Kabir F, Lima A, Babji S, Shrestha SK, Mason
CJ, Kalam A, Bessong P, Ahmed T, Mduma E, Bhutta ZA, Lima I, Ramdass R, Moulton LH, Lang D, George A, Zaidi AKM, Kang G, Houpt ER, Kosek MN, MAL-ED Network. Astrovirus infection and diarrhea in 8 countries. Pediatrics. 2018;141(1):e20171326. https://doi.org/10.1542/peds.2017-1326.

23. Biscaro V, Piccinelli G, Gargiulo F, laniro G, Caruso A, Caccuri F, De Francesco MA. Detection and molecular characterization of enteric viruses in children with acute gastroenteritis in Northern Italy. Infect Genet Evol. 2018;60:35-41. https://doi.org/10.1016/j.meegid.2018.02.011.

24. Varela MF, Rivadulla E, Lema A, Romalde JL. Human sapovirus among outpatients with acute gastroenteritis in Spain: a 1-year study. Viruses. 2019;11(2):144. https://doi.org/10.3390/v11020144.

25. Pativada M, Nataraju SM, Ganesh B, Rajendran K, Ramamurthy T, Ganguly S, Bhattacharya MK, Ghosh M, Kobayashi N, Krishnan T. Emerging trends in the epidemiology of human astrovirus infection among infants, children and adults hospitalized with acute watery diarrhea in Kolkata, India. Infect Genet Evol. 2012;12(8):1685-93. https://doi.org/10.1016/j.meegid. 2012.07.018.

26. Mustafa H, Palombo EA, Bishop RF. Epidemiology of astrovirus infection in young children hospitalized with acute gastroenteritis in Melbourne, Australia, over a period of four consecutive years, 1995 to 1998. J Clin Microbiol. 2000;38(3):1058-62. https://doi.org/10.1128/JCM.38.3.10581062.2000.

27. Phan TG, Okame M, Nguyen TA, Nishio O, Okitsu S, Ushijima H. Genetic diversity of sapovirus in fecal specimens from infants and children with acute gastroenteritis in Pakistan. Arch Virol. 2005;150(2):371-7. https://doi. org/10.1007/s00705-004-0401-0.

28. Phan TG, Nguyen TA, Nishimura S, Nishimura T, Yamamoto A, Okitsu S, Ushijima $\mathrm{H}$. Etiologic agents of acute gastroenteritis among Japanese infants and children: virus diversity and genetic analysis of sapovirus. Arch Virol. 2005;150(7):1415-24. https://doi.org/10.1007/s00705-005-0514-0.

29. Oka T, Wang Q, Katayama K, Saif $L J$. Comprehensive review of human sapoviruses. Clin Microbiol Rev. 2015;28(1):32-53. https://doi.org/10. 1128/CMR.00011-14.

30. Tarr GAM, Downey E, Pang XL, Zhuo R, Strickland AJ, Ali S, Lee BE, Chui L, Tarr PI, Freedman SB. Clinical profiles of childhood astrovirus-, sapovirus-, and norovirus-associated acute gastroenteritis in pediatric emergency departments in Alberta, 2014-2018. J Infect Dis. 2021. https://doi.org/10. 1093/infdis/jiab429.

31. Taylor MB, Marx FE, Grabow WO. Rotavirus, astrovirus and adenovirus associated with an outbreak of gastroenteritis in a South African child care centre. Epidemiol Infect. 1997;119(2):227-30. https://doi.org/10. 1017/s0950268897007814.

32. Román E, Wilhelmi I, Colomina J, Villar J, Luz Cilleruelo M, Nebreda V, Del Alamo M, Sánchez-Fauquier A. Acute viral gastroenteritis: proportion and clinical relevance of multiple infections in Spanish children. J Med Microbiol. 2003;52(Pt 5):435-40. https://doi.org/10.1099/jmm.0.05079-0.

33. Nadan S, Taylor MB, Groome MJ, Cohen C, Madhi SA, Page NA. Epidemiology of human astroviruses among children younger than 5 years: prospective hospital-based sentinel surveillance in South Africa, 2009-2014. J Med Virol. 2019;91 (2):225-34. https://doi.org/10.1002/jmv.25308.

34. Gupta S, Krishnan A, Sharma S, Kumar P, Aneja S, Ray P. Changing pattern of prevalence, genetic diversity, and mixed infections of viruses associated with acute gastroenteritis in pediatric patients in New Delhi, India. J Med Virol. 2018;90(3):469-76. https://doi.org/10.1002/jmv.24980.

35. Zaraket H, Abou-El-Hassan H, Kreidieh K, Soudani N, Ali Z, Hammadi M, Reslan L, Ghanem S, Hajar F, Inati A, Rajab M, Fakhouri H, Ghanem B, Baasiri G, Melhem NM, Dbaibo G. Characterization of astrovirus-associated gastroenteritis in hospitalized children under five years of age. Infect Genet Evol. 2017;53:94-9. https://doi.org/10.1016/j.meegid.2017.05.016.

36. Xavier Mda P, Carvalho Costa FA, Rocha MS, Andrade Jda S, Diniz FK, Andrade TR, Miagostovich MP, Leite JP, Volotão EM. Surveillance of human astrovirus infection in Brazil: the first report of MLB1 astrovirus. PLOS ONE. 2015;10(8):e0135687. https://doi.org/10.1371/journal.pone.0135687.

37. Liu MQ, Yang BF, Peng JS, Zhou DJ, Tang L, Wang B, Liu Y, Sun SH, Ho WZ. Molecular epidemiology of astrovirus infection in infants in Wuhan, China. J Clin Microbiol. 2007;45(4):1308-9. https://doi.org/10.1128/JCM. 00010-07.

38. Medina SM, Gutierrez MF, Liprandi F, Ludert JE. Identification and type distribution of astroviruses among children with gastroenteritis in Colombia and Venezuela. J Clin Microbiol. 2000;38(9):3481-3. https://doi.org/10. 1128/JCM.38.9.3481-3483.2000. 
39. Diez-Valcarce M, Castro CJ, Marine RL, Halasa N, Mayta H, Saito M, Tsaknaridis L, Pan CY, Bucardo F, Becker-Dreps S, Lopez MR, Magaña LC, Ng TFF, Vinjé J. Genetic diversity of human sapovirus across the Americas. J Clin Virol. 2018;104:65-72. https://doi.org/10.1016/j.jcv.2018.05.003.

\section{Publisher's Note}

Springer Nature remains neutral with regard to jurisdictional claims in published maps and institutional affiliations.

- fast, convenient online submission

- thorough peer review by experienced researchers in your field

- rapid publication on acceptance

- support for research data, including large and complex data types

- gold Open Access which fosters wider collaboration and increased citations

- maximum visibility for your research: over 100M website views per year

At $\mathrm{BMC}$, research is always in progress.

Learn more biomedcentral.com/submissions 